\title{
Yarı-asal Halkalarda Ortogonal Yarı-türevler Üzerine
}

\author{
Öznur GÖLBAŞI ${ }^{1 *}$, Fatih BILGIIN ${ }^{1}$ \\ ${ }^{1}$ Cumhuriyet University, Institute of Science, Department of Mathematics, 58140 Sivas, Turkey
}

Received: 25.02.2016; Accepted: 06.04.2016

\begin{abstract}
Özet. Bu makalede, " $R$ bir 2 -torsion free yarı-asal halka, $f$ ve $h, g$ örten fonksiyonu ile belirlenmiş sıfirdan farklı iki yarıtürev olmak üzere $f$ ve $h$ in ortogonal olması için gerek ve yeter koşul i) $f h=0$, ii) $f h+h f=0$, iii) her $x \in R$ için $f(x) h(x)=0$, iv) $f h, g^{2}$ fonksiyonu ile belirlenmiş bir yarı-türevdir, koşullarından herhangi birinin sağlanmasıdır" ve ayrıca "her $x, y \in R$ için i) $f(x y)=f(x) f(y)$, ii) $f(x y)=f(y) f(x)$, iii) $f(x y) \pm x y \in Z$ koşullarından birini sağlayan $R$ halkası sıfirdan farklı bir merkezil ideal kapsar" teoremleri gösterilmiştir.
\end{abstract}

Anahtar Kelimeler: Yar1-türevler, ortogonal türevler, yar1-asal halkalar

\section{On The Orthogonal Semi Derivatives in Semi Principal Rings}

Abstract. In this paper, it is shown that if $R$ is 2 -torsion free semiprime ring, $f$ and $h$ are semiderivations associated with a surjective function $g$ of $R, f$ and $h$ are orthogonal semiderivations if and only if the following conditions are satisfied i) $f h=0$, ii) $f h+h f=0$, iii) $\forall x \in R, f(x) h(x)=0$, iv) $f h$ is a semiderivation associated with a $g^{2}$ function. Also, it is proved that if i) $f(x y)=f(x) f(y)$, ii) $f(x y)=f(y) f(x)$, iii) $f(x y) \pm x y \in Z$ conditions are satisfied for all $x, y \in R$, then $R$ contains a nonzero central ideal.

Keywords: Semi-derivations, orthogonal derivations, semi-prime rings

\section{GíRIş}

Halkalarda türev tanımı ilk olarak 1957 yılında E. C. Posner tarafından yapılan bir çalışmada verilmiştir [10]. $R$ bir halka, $d: R \rightarrow R$ toplamsal bir dönüşüm olsun. Eğer her $x, y \in R$ için

$$
d(x y)=d(x) y+x d(y)
$$

koşulu sağlanıyor ise $d$ ye $R$ halkasının bir türevi denir.

E. C. Posner bu makalesinde türev yardımıyla bir asal halkanın değişmeli olma koşullarını incelemiş ve konuyla ilgili çalışmalara bir ön kaynak oluşturmuştur. Türevler teorisinin gelişimiyle beraber halka üzerinde farklı türev tanımları yapılmış, asal ve yarı-asal halkalarda bu türevlerin sağladığı özellikler pek çok araştırmacı tarafından incelenmiş̧ir. Son altmış yıldır türevler üzerinde yapılan bu çalışmalarda halkaların uygun bir alt kümesi veya farklı türevleri alınarak değişmeli olma koşulları genelleştirilmiştir.

1983 yılında J. Bergen tarafından halkalarda yarı-türev tanımı verilmiştir [5]. $R$ bir halka, $d: R \rightarrow$ $R$ toplamsal bir dönüşüm ve $g: R \rightarrow R$ herhangi bir dönüşüm olsun. Eğer her $x, y \in R$ için

$$
\begin{aligned}
& f(x y)=f(x) g(y)+x f(y)=f(x) y+g(x) f(y) \\
& f(g(x))=g(f(x))
\end{aligned}
$$

\footnotetext{
* Corresponding author. Email address: ogolbasi@ cumhuriyet.edu.tr 


\section{GÖLBAŞI, BİLGİN}

koşulu sağlanıyor ise $f$ ye $R$ halkasında $g$ fonksiyonu ile belirlenmiş bir yarı-türev denir.

J. Bergen bu çalışmasında asal halkaların yarı-türevlerinin cebirsel özelliklerini incelemiştir. Bu çalışmadan sonra türevli asal ve yarı-asal halkalar için elde edilmiş olan bazı sonuçlar yarı-türevler için araştırımıştır. 1984 yılında $\mathrm{J}$. C. Chang, $g$ ile belirlenmiş bir $f$ yarı-türevi için eğer $R$ asal halka ise $g$ nin bir halka homomorfizması olduğunu göstermiştir [8]. Chang bu makalesinde türevle ilgili değişmeli olma koşullarının yarı-türevler için sağlandığı ispatlamıştır. 1988 yılında H. E. Bell ve W. S. Martindale ise bu teoremleri yarı-türevler ve $R$ halkasının sıfırdan farklı bir ideali için araştırmışlardır [3].

1989 yılında M. Bresar ve J. Vukman tarafindan halkalarda ortogonal türev tanımı verilmiştir [6]. $R$ bir halka, $f$ ve $h$ sıfirdan farklı iki türev olsun. Eğer her $x, y \in R$ için

$$
d(x) R h(y)=(0)=h(y) R d(x)
$$

koşulu sağlanıyor ise $d$ ve $h$ ortogonal türevlerdir denir. Yazarlar bu çalışmalarında $R, 2$-torsion free bir yar1-asal halka, $f$ ve $h$ sıfirdan farklı iki türev olmak üzere, $f$ ve $h$ ortogonaldir ancak ve ancak aşağıdaki koşullar sağlanır:

i) $f h=0$

ii) $f h+h f=0$

iii) Her $x \in R$ için $f(x) h(x)=0$

iv) Her $x \in R$ için $f(x) h(x)+h(x) f(x)=0$

v) $f h$ bir türevdir.

teoremini ispatlamışlardır. Bu teorem daha sonra pek çok araştırmacı tarafından halkanın idealleri veya farklı türevleri alınarak genelleştirilmiştir.

Öte yandan 1989 yılında H. E. Bell ve L. C. Kappe tarafından $R$ yarı-asal halkasında verilen bir $d$ türevi için eğer $d, R$ nin sıfirdan farklı bir sağ ideali üzerinde homomorfizm veya anti-homomorfizm ise bu durumda $d=0$ olduğu gösterilmiştir [4].

M. Ashraf ve N. Rehman 2001 yılında $R$ bir asal halka, $I, R$ halkasının sıfırdan farklı bir ideali ve $d, R$ üzerinde bir türev olmak üzere her $x, y \in I$ için $d(x y) \pm x y \in Z$ ise $I \subseteq Z$ olduğunu ispatlamışlardır [1]. 2007 yılında, M. Ashraf, A. Asma ve A. Shakir ise $f: R \rightarrow R$ bir genelleştirilmiş türev olmak üzere her $x, y \in R$ için $f(x y) \pm x y \in Z$ koşulu sağlanıyorsa $R$ halkasının değişmeli olduğunu göstermişlerdir [2]. Bu teoremler de pek çok matematikçi tarafından araştırılmıştır.

Bu makalede $R$ bir 2 -torsion free yar1-asal halka, $f$ ve $h, g$ örten fonksiyonu ile belirlenmiş sıfırdan farklı iki yarı-türev olmak üzere $f$ ve $h$ in ortogonal olması için gerek ve yeter kosul i) $f h=0$, ii) $f h+h f=0$, iii) her $x \in R$ için $f(x) h(x)=0$, iv) $f h, g^{2}$ fonksiyonu ile belirlenmiş bir yar1türevdir, koşullarından herhangi birinin sağlanmasıdır ve ayrıca her $x, y \in R$ için i) $f(x y)=f(x) f(y)$, ii) $f(x y)=f(y) f(x)$, iii) $f(x y) \pm x y \in Z$ koşullarından birini sağlayan $R$ halkası sıfırdan farklı bir merkezil ideal kapsar, teoremleri gösterilmiştir.

\section{SONUÇLAR}

Tanım 2.1: $R$ bir halka, $f, h: R \rightarrow R$ tanımlı $g$ (örten) fonksiyonu ile belirlenmiş iki yarı-türev olsun. Ĕger her $x, y \in R$ için 


$$
f(x) R h(y)=(0)=h(y) R f(x)
$$

koşulu sağlanıyor ise $f$ ve $h$ ortogonal türevlerdir denir.

Lemma 2.1[6, Lemma 1]: $R, 2$-torsion free bir yar1-asal halka, $a, b \in R$ olsun. Eğer her $x \in R$ için

i) $a x b=0$

ii) $b x a=0$

iii) $a x b+b x a=0$

koşulları birbirine denktir. Üstelik bu koşullardan herhangi biri sağlanıyorsa bu durumda $a b=b a=0$ dir.

Lemma 2.2[6, Lemma 2]: $R$ bir yarı-asal halka, $f, h: R \rightarrow R$ tanımlı iki toplamsal dönüşüm olsun. Eğer her $x \in R$ için $f(x) R h(x)=(0)$ ise bu durumda her $x, y \in R$ için $f(x) R h(y)=(0)$ sağlanır.

Lemma 2.3[9, Lemma 2.1]: $R$ bir yarı-asal halka, $I, R$ halkasının sıfırdan farklı bir ideali ve $f, g$ örten fonksiyonu ile belirlenmiş sıfırdan farklı bir yarı-türevi olsun. Ĕger her $x, y \in I$ için $[f(x), x]=0$ ise bu durumda $R$ sıfırdan farklı bir merkezil ideal kapsar.

Lemma 2.4: $R, 2$-torsion free bir yarı-asal halka, $f, h: R \rightarrow R$ tanımlı $g$ fonksiyonu ile belirlenmiş iki yarı-türev olsun. Bu durumda $f$ ve $g$ ortogonaldir. $\Leftrightarrow \operatorname{Her} x, y \in R$ için $f(x) h(y)+h(x) f(y)=0$ dır.

$$
\text { İspat: } \Rightarrow \operatorname{Her} x, y \in R \text { için }
$$

$$
f(x) h(y)+h(x) f(y)=0
$$

sağlansın. (2.1) eşitliğinde $y$ yerine $y x$ yazılırsa

$$
\begin{aligned}
& 0=f(x) h(y x)+h(x) f(y x) \\
& =f(x)(h(y) g(x)+y h(x))+h(x)(f(y) g(x)+y f(x)) \\
& =f(x) h(y) g(x)+f(x) y h(x)+h(x) f(y) g(x)+h(x) y f(x) \\
& =(f(x) h(y)+h(x) f(y)) g(x)+f(x) y h(x)+h(x) y f(x)
\end{aligned}
$$

bulunur. Bu ifadede (2.1) eşitliği kullanılırsa

$$
f(x) y h(x)+h(x) y f(x)=0, \forall x, y \in R
$$

elde edilir. (2.2) eşitliğine Lemma 2.1 uygulanırsa

$$
f(x) y h(x)=0, \forall x, y \in R
$$

bulunur. Burada $f$ ve $h$ toplamsal dönüşümler olduğu için Lemma 2.2 den

$$
f(x) y h(z)=0, \forall x, y, z \in R
$$

dir. Yani

$$
f(x) \operatorname{Rh}(z)=(0), \forall x, z \in R
$$




\section{GÖLBAŞI, BİLGİN}

elde edilir. Yine (2.2) eşitliğinde Lemma 2.1 den

$$
h(x) y f(x)=0, \forall x, y \in R
$$

olur. Lemma 2.2 den ise

$$
h(x) R f(z)=(0), \forall x, z \in R
$$

bulunur. Böylece bu son iki eşitlikten

$$
f(x) R h(z)=(0)=h(x) R f(z), \forall x, z \in R
$$

dir. Bu durumda $f$ ve $h$ ortogonaldir.

$\Leftarrow: f$ ve $h$ ortogonal yarı-türevler olsun. Bu durumda ortogonal olma tanımından

$$
f(x) R h(z)=(0)=h(x) R f(z), \forall x, z \in R
$$

sağlanır. $f(x) R h(z)=0$ eşitliğine Lemma 2.1 uygulanarak

$$
f(x) h(z)=0=h(z) f(x), \forall x, z \in R
$$

elde edilir. Böylece her $x, z \in R$ için $f(x) h(z)+h(z) f(x)=0$ sağlanır.

NOT: $f, h: R \rightarrow R$ tanımlı $g$ fonksiyonu ile belirlenmiş iki yarı-türev olsun. Bu durumda her $x, y \in R$ için

$$
\begin{aligned}
& (f h)(x y)=f(h(x y))=f(h(x) g(y)+x h(y)) \\
& =(f h)(x) g^{2}(y)+h(x)(f g)(y)+f(x)(g h)(y)+x(f h)(y)
\end{aligned}
$$

olur. Buradan her $x, y \in R$ için

$$
(f h)(x y)=(f h)(x) g^{2}(y)+h(x)(f g)(y)+f(x)(g h)(y)+x(f h)(y)
$$

dir.

Teorem 2.1: $R, 2$-torsion free bir yarı-asal halka, $f$ ve $h, g$ örten fonksiyonu ile belirlenmiş sıfırdan farklı iki yarı-türev olsun. Bu durumda $f$ ve $h$ ortogonaldir ancak ve ancak aşağıdaki koşullar sağlanır:

i) $f h=0$

ii) $f h+h f=0$

iii) Her $x \in R$ için $f(x) h(x)=0$

iv) $f h, g^{2}$ fonksiyonu ile belirlenmiş bir yarı-türevdir.

İspat: $\mathrm{i}) \Rightarrow f$ ve $h$ ortogonal mi?

$$
f h=0
$$

olduğunu kabul edelim. Bu durumda her $x, y \in R$ için

$$
(f h)(x y)=0
$$


sağlanır. Bu ifade (2.3) eşitliği kullanılarak düzenlenirse

$$
0=(f h)(x y)=(f h)(x) g^{2}(y)+h(x)(f g)(y)+f(x)(g h)(y)+x(f h)(y)
$$

bulunur. Bu eşitlikte $f h=0$ olduğu kullanılırsa her $x, y \in R$ için

$$
h(x)(f g)(y)+f(x)(g h)(y)=0
$$

elde edilir. $h, g$ ile belirlenmiş yarı-türev olduğu için $h g=g h$ sağlanır. Böylece (2.4) eşitliği

$$
h(x)(f g)(y)+f(x)(h g)(y)=0
$$

olur. Fonksiyonlarda bileşke tanımından son eşitlik

$$
h(x) f(g(y))+f(x) h(g(y))=0
$$

biçiminde yazılır. $g$ fonksiyonu örten fonksiyon olduğundan $g(y)=z, \forall y, z \in R$ yazılabilir. O halde son eşitlikten

$$
h(x) f(z)+f(x) h(z)=0, \forall x, z \in R
$$

elde edilir. Böylece Lemma $2.1 \operatorname{den} f$ ve $h$ ortogonaldir.

$f$ ve $h$ ortogonaldir $\Rightarrow$ i) $\mathrm{mi}$ ?

$f$ ve $h$ ortogonal yarı-türevler olsun. Bu durumda tanım gereği

$$
f(x) R h(z)=(0), \forall x, z \in R
$$

sağlanır. Dolayısıyla

$$
f(x) y h(z)=0, \forall x, y, z \in R
$$

olur. Böylece Lemma 2.1 den

$$
f(x) h(z)=0, \forall x, z \in R
$$

bulunur.

Şimdi $f(x) y h(z)=0$ olduğu için

$$
\begin{aligned}
& 0=f(f(x) y h(z))=f^{2}(x) y h(z)+(g f)(x) f(y h(z)) \\
& =f(f(x)) y h(z)+(g f)(x) f(y)(g h)(z)+(g f)(x) y(f h)(z)
\end{aligned}
$$

sağlanır. Bu ifadede (2.5) eşitliği kullanılarak

$$
(g f)(x) f(y)(g h)(z)+(g f)(x) y(f h)(z)=0, \forall x, y, z \in R
$$

bulunur. $f$ ve $h, g$ ile belirlenmiş yarı-türevler olduğu için $f g=g f, h g=g h$ sağlanır. Bu ifadeler son eşitlikte kullanılırsa

$$
(g f)(x) f(y)(h g)(z)+(f g)(x) y(f h)(z)=0
$$

dır. Yani 


\section{GÖLBAŞI, BİLGINN}

$$
g(f(x)) f(y) h(g(z))+f(g(x)) y f(h(z))=0, \forall x, y, z \in R
$$

olur. Bu ifadenin ilk terimi $\forall x, z \in R$ için $f(x) h(z)=0$ olduğundan sıfırdır. Böylece

$$
f(g(x)) y f(h(z))=0, \forall x, y, z \in R
$$

elde edilir. Yine $g$ örten fonksiyon olduğu için

$$
f(t) y f(h(z))=0, \forall y, z, t \in R
$$

sağlanır. Bu eşitlikte $t$ yerine $h(z)$ yazılırsa

$$
f(h(z)) y f(h(z))=0, \forall y, z \in R
$$

olur. Yani

$$
f(h(z)) R f(h(z))=(0), \forall z \in R
$$

dir. $R$ yarı-asal halka olduğu için

$$
f(h(z))=(0), \forall z \in R
$$

ve dolayısıyla $f h=0$ bulunur. İspat tamamlanır.

ii) $\Rightarrow f$ ve $h$ ortogonal mi?

Hipotezden $f h+h f=0$ sağlansın. Bu durumda her $x, y \in R$ için

$$
(f h+h f)(x y)=0
$$

sağlanır. Bu ifade $f$ ve $h$ in $g$ ile belirlenen yarı-türevler ve $R$ nin bir 2 -torsion free halka olduğu kullanılarak düzenlenirse

$$
f(x) h(g(y))+h(x) f(g(y))=0, \forall x, y \in R
$$

bulunur. Yine $g$ örten fonksiyon olduğu için son eşitlikten

$$
f(x) h(z)+h(x) f(z)=0, \forall x, z \in R
$$

elde edilir. Bu ifadede Lemma 2.4 kullanılırsa $f$ ve $h$ ortogonal yar1-türevlerdir bulunur.

$f$ ve $h$ ortogonaldir $\Rightarrow$ ii) $\mathrm{mi}$ ?

$f$ ve $h$ ortogonal yarı-türevler olsun. Bu durumda bu teoremin (i) koşulu sağlanır. Dolayısıyla $f h=0, h f=0$ olur Böylece $f h+h f=0$ elde edilir.

iii) $\Rightarrow f$ ve $h$ ortogonal mi?

(iii) hipotezinden

$$
f(x) h(x)=0, \forall x \in R
$$

sağlanır. (2.6) eşitliğnde $x$ yerine $x+y, y \in R$ yazılır ve hipotez kullanılırsa

$$
f(x) h(y)+f(y) h(x)=0, \forall x, y \in R
$$


elde edilir. (2.7) eşitliğinde $y$ yerine $y z, z \in R$ yazılırsa

$$
\begin{array}{r}
\begin{aligned}
0= & f(x) h(y z)+f(y z) h(x) \\
= & f(x)(h(y) g(z)+y h(z))+(f(y) g(z)+y f(z)) h(x) \\
= & f(x) h(y) g(z)+f(x) y h(z)+f(y) g(z) h(x)+y f(z) h(x)
\end{aligned}
\end{array}
$$

olur. Öte yandan (2.7) eşitliğinden

$$
f(x) h(y)=-f(y) h(x), f(z) h(x)=-f(x) h(z)
$$

sağlanır. Bu eşitlikler son ifadede kullanılarak

$$
[f(x), y] h(z)+f(y)[g(z), h(x)]=0, \forall x, y, z \in R
$$

bulunur. (2.8) eşitliğinde $y$ yerine $f(x)$ yazılırsa

$$
f^{2}(x)[g(z), h(x)]=0, \forall x, y, z \in R
$$

olur. $g$ örten fonksiyon olduğu için son eşitlikten

$$
f^{2}(x)[t, h(x)]=0, \forall x, y, t \in R
$$

bulunur. Bu eşitlikte $t$ yerine $t r, r \in R$ yazılır ve bu eşitlik kullanılırsa

$$
f^{2}(x) R[r, h(x)]=(0), \forall x, r \in R
$$

olur. $I: R \rightarrow R, I(x)=[r, x]$ dönüşümü toplamsal bir dönüşümdür. Bu nedenle son eşitlikte Lemma (2.2) kullanilarak

$$
f^{2}(x) R[r, h(y)]=(0), \forall x, y, r \in R
$$

elde edilir. Yine bu son eşitlikte $x$ yerine $x u, u \in R$ yazılır ve bu eşitlik ile $g f=f g$ olduğu kullanılırsa, $R, 2$-torsion free halka olduğu için

$$
f(x) f(t) z[r, h(y)]=0, \forall x, y, z, r, t \in R
$$

bulunur. Burada $x$ yerine $x w, w \in R$ yazılarak benzer işlemlerle

$$
f(x) w f(t) z[r, h(y)]=0, \forall x, y, z, r, t, w \in R
$$

dir. Yani

$$
f(x) R f(t) R[r, h(y)]=(0), \forall x, y, r, t \in R
$$

olur. Bu eşitlikten özel olarak

$$
f(x) R[r, h(y)] R f(x) R[r, h(y)]=(0)
$$

sağlanır. Böylece $R$ bir yarı-asal halka olduğundan son eşitlikten $f(x) R[r, h(y)]=(0)$ elde edilir. Böylece

$$
f(x) z[r, h(y)]=0, \forall x, y, z, r \in R
$$




\section{GÖLBAŞI, BİLGİN}

olur. Burada $r$ yerine $f(x)$ yazalım. Bu durumda

$$
f(x) z[f(x), h(y)]=0, \forall x, y, z \in R
$$

sağlanır. Yine burada $z$ yerine $h(y) z$ yazılırsa

$$
f(x) h(y) z[f(x), h(y)]=0, \forall x, y, z \in R
$$

bulunur. Öte yandan (2.9) eşitliği soldan $h(y)$ ile çarpılır ve burada $r$ yerine $f(x)$ yazılırsa

$$
h(y) f(x) z[f(x), h(y)]=0, \forall x, y, z, r \in R
$$

elde edilir. Böylece son iki eşitlik birbirinden çıkarılıp düzenlenirse

$$
[f(x), h(y)] R[f(x), h(y)]=(0), \forall x, y \in R
$$

sağlanır. Bu durumda $R$ bir yarı-asal halka olduğundan son eşitlikten

$$
[f(x), h(y)]=0, \forall x, y \in R
$$

bulunur. Böylece

$$
f(x) h(y)=h(y) f(x), \forall x, y \in R
$$

dir. Şimdi (2.7) eşitliğinde bu ifade kullanılırsa

$$
f(x) h(y)+h(x) f(y)=0, \forall x, y \in R
$$

elde edilir. Bu durumda Lemma $2.4 \operatorname{den} f$ ve $h$ ortogonal yar1-türevlerdir.

$f$ ve $h$ ortogonaldir $\Rightarrow$ iii) $\mathrm{mi}$ ?

$f$ ve $h$ ortogonal yar1-türevler olsun. Bu durumda tanımdan $f(x) R h(y)=(0), \forall x, y \in R$ sağlanır. Böylece Lemma 2.3 den $f(x) h(y)=(0), \forall x, y \in R$ olur. Özel olarak $y$ yerine $x$ yazılırsa $f(x) R h(x)=(0), \forall x \in R$ elde edilir. İspat tamamlanır.

iv) $\Rightarrow f$ ve $h$ ortogonal mi?

(iv) hipotezinden $f h, g^{2}$ ile belirlenen bir yarı-türev olsun. Bu durumda

$$
(f h)(x y)=(f h)(x) g^{2}(y)+x(f h)(y)=0, \forall x, y \in R
$$

olur. Öte yandan

$$
\begin{aligned}
& f h(x y)=f(h(x y))=f(h(x) g(y)+x h(y)) \\
& =(f h)(x) g^{2}(y)+h(x)(f g)(y)+f(x)(g h)(y)+x(f h)(y)
\end{aligned}
$$

dir. Bu son iki eşitlikten her $x, y \in R$ için

$$
h(x)(f g)(y)+f(x)(g h)(y)=0
$$

elde edilir. $g h=h g$ olduğu kullanılarak

$$
h(x) f(g(y))+f(x) h(g(y))=0
$$


yazılır. $g$ örten fonksiyon olduğu için son ifadeden

$$
h(x) f(z)+f(x) h(z)=0, \forall x, z \in R
$$

ulaşılır. Böylece Lemma 2.4 den $f$ ve $h$ ortogonal yarı-türevlerdir.

$f$ ve $h$ ortogonaldir $\Rightarrow$ iv) mi?

$f$ ve $h$ ortogonal yarı-türevler olsun. Bu durumda bu teoremin (i) koşulu sağlanır. Dolayısıyla $f h=0$ olur. Üstelik sıfır dönüşümü bir yarı-türev olduğu için $f h$ da bir yar1-türev olur ve ispat biter.

Tanım 2.2: $R$ bir halka, $S, R$ nin boştan farklı bir alt kümesi ve $f: R \rightarrow R$ bir fonksiyon olsun. Eğer $\forall x, y \in S$ için

$$
f(x y)=f(x) f(y)
$$

koşulu sağlanıyor ise $f$ ye $S$ üzerinde bir homomorfizm denir.

Tanım 2.3: $R$ bir halka, $S, R$ nin boştan farklı bir alt kümesi ve $f: R \rightarrow R$ bir fonksiyon olsun. Eğer $\forall x, y \in \mathrm{S}$ için

$$
f(x y)=f(y) f(x)
$$

koşulu sağlanıyor ise $f$ ye $S$ üzerinde bir anti-homomorfizm denir.

Teorem 2.2: $R$ bir yarı-asal halka, $f, g$ örten fonksiyonu ile belirlenmiş sıfırdan farklı bir yarı-türev olsun. Ĕger $f, R$ üzerinde bir homomorfizma ise bu durumda $R$ halkası sıfırdan farklı merkezil bir ideal kapsar.

İspat: $f, R$ üzerinde bir homomorfizma olsun. Bu durumda her $x, y, z \in R$ için

$$
f(x y)=f(x) f(y)=f(x) y+g(x) f(y)
$$

sağlanır. Bu eşitlikte $y$ yerine $y z, z \in R$ yazılırsa

$$
f(x) y z+g(x) f(y z)=f(x) f(y z)=f(x) f(y) f(z)
$$

olur. Bu eşitliğin sağ tarafinda $f$ in $R$ üzerinde bir homomorfizma olduğu kullanılırsa

$$
f(x) y z+g(x) f(y z)=f(x y) f(z)
$$

elde edilir. $f$ yarı-türev olduğundan

$$
f(x) y z+g(x) f(y z)=f(x) y f(z)+g(x) f(y) f(z)
$$

bulunur. Yine bu eşitliğin sağ tarafı için $f, R$ üzerinde bir homomorfizma olduğundan

$$
f(x) y z+g(x) f(y z)=f(x) y f(z)+g(x) f(y z)
$$

dir. Böylece

$$
f(x) y z=f(x) y f(z)
$$

olur. Yani 


\section{GÖLBAŞI, BİLGİN}

$$
f(x) y(z-f(z))=0, \forall x, y, z \in R
$$

elde edilir. Bu eşitlikte $y$ yerine $f(y)$ yazılırsa, bu durumda

$$
f(x) f(y)(z-f(z))=0, \forall x, y, z \in R
$$

olur. Bu son eşitlikte hipotez kullanılarak

$$
f(x y)(z-f(z))=0, \forall x, y, z \in R
$$

sağlanır. Bu eşitlikten $f$ yarı-türev olduğu için

$$
f(x) g(y)(z-f(z))+x f(y)(z-f(z))=0
$$

bulunur. Bu ifadede $g$ nin örten olduğu ve (2.10) eşitliği kullanılırsa

$$
x f(y)(z-f(z))=0, \forall x, y, z \in R
$$

elde edilir. Yani

$$
x f(y) z=x f(y) f(z)
$$

dir. Yine hipotezden

$$
x f(y) z=x f(y z)
$$

ve $f$ yar1-türev olduğundan

$$
x f(y) z=x f(y) z+x g(y) f(z)
$$

olur. Böylece

$$
x g(y) f(z)=0, \forall x, y, z \in R
$$

elde edilir. $g$ örten fonksiyon olduğu için son ifadeden

$$
\operatorname{xrf}(z)=0, \forall x, r, z \in R
$$

bulunur. Bu eşitlik soldan $r f(z)$ ile çarpılır ve $R$ nin yar1-asal halka olduğu kullanılırsa

$$
r f(z)=0, \forall r, z \in R
$$

olur. Şimdi (2.11) eşitliği soldan $f(z)$ ve sağdan $x$ ile çarpılırsa

$$
f(z) \operatorname{xr} f(z) x=0, \forall x, r, z \in R
$$

olur. $R$ halkasının yarı-asallığı kullanılarak

$$
f(z) x=0, \forall x, z \in R
$$

elde edilir. Böylece (2.12) ve (2.13) eşitliklerinden

$$
x f(z)=f(z) x, \forall x, z \in R
$$

sağlanır. Özel olarak $x f(x)=f(x) x$, yani 


$$
[f(x), x]=0, \forall x \in R
$$

olur. Böylece Lemma 2.3 den $R$ halkası sıfırdan farklı merkezil bir ideal kapsar.

Teorem 2.3: $R$ bir yarı-asal halka, $f, g$ örten fonksiyonu ile belirlenmiş sıfırdan farklı bir yarı-türev olsun. Ĕger $f, R$ üzerinde bir anti-homomorfizma ise bu durumda $R$ halkası sıfırdan farklı merkezil bir ideal kapsar.

İspat: $f, R$ üzerinde bir anti-homomorfizma olsun. Bu durumda her $x, y, z \in R$ için

$$
f(x y)=f(y) f(x)=f(x) y+g(x) f(y)
$$

sağlanır. Bu eşitlikte $y$ yerine $x y$ yazılırsa

$$
f(x) x y+g(x) f(x y)=f(x y) f(x)
$$

olur. Bu eşitliğin sağ tarafında $f$ in $R$ üzerinde bir yarı-türev olduğu kullanılırsa

$$
f(x) x y+g(x) f(x y)=f(x) y f(x)+g(x) f(y) f(x)
$$

dir. Yine $f, R$ üzerinde bir anti-homomorfizma olduğundan

$$
f(x) x y+g(x) f(x y)=f(x) y f(x)+g(x) f(x y)
$$

bulunur. Böylece

$$
f(x) x y=f(x) y f(x), \forall x, y \in R
$$

elde edilir. Bu eşitlikte $y$ yerine $y x$ yazılır ve tekrar bu eşittlik kullanılırsa

$$
f(x) y f(x) x=f(x) y x f(x), \forall x, y \in R
$$

elde edilir. Yani

$$
f(x) y[f(x), x]=0, \forall x, y \in R
$$

bulunur. Burada $y$ yerine $x y$ yazılarak

$$
f(x) x y[f(x), x]=0, \forall x, y \in R
$$

sağlanır. (2.14) e eşitliği soldan $x$ ile çarpılırsa

$$
x f(x) y[f(x), x]=0, \forall x, y \in R
$$

olur. Bu iki eşitlik birbirinden çıkarılarak düzenlenirse

$$
[f(x), x] y[f(x), x]=0, \forall x, y \in R
$$

elde edilir. R yar1-asal halka olduğu için

$$
[f(x), x]=0, \forall x \in R
$$

olur. Böylece Lemma 2.3 den $R$ halkası sıfırdan farklı merkezil bir ideal kapsar. 


\section{GÖLBAŞI, BİLGİN}

Teorem 2.4: $R, 2$-torsion free bir yarı-asal halka, $f, g$ örten fonksiyonu ile belirlenmiş sıfırdan farklı bir yar1-türev olsun. Eğer her $x, y \in R$ için $f(x y) \pm x y \in Z$ ise bu durumda $R$ halkası sıfırdan farkl1 merkezil bir ideal kapsar.

İspat: Hipotezden her $x, y \in R$ için

$$
f(x y) \pm x y \in Z
$$

sağlansın. Bu eşitlikte $y$ yerine $y z, z \in R$ yazılırsa $f(x y z) \pm x y z \in Z$ olur. Bu ifade $f$ in $R$ üzerinde bir yar1-türev olduğu kullanılarak düzenlenirse her $x, y, z \in R$ için

$$
(f(x y) \pm x y) z+g(x y) f(z) \in Z
$$

bulunur. Bu eşitlik $z \in R$ ile komüte edilir ve $f(x y) \pm x y \in Z$ olduğu kullanılırsa

$$
[g(x y) f(z), z]=0, \forall x, y, z \in R
$$

olur. $g$ örten fonksiyon olduğu için

$$
[r f(z), z]=0, \forall r, z \in R
$$

elde edilir. Bu eşitlikte $r$ yerine $f(z) r$ yazılır ve bu eşitlik tekrar kullanılırsa, bu durumda

$$
[f(z), z] r f(z)=0, \forall r, z \in R
$$

elde edilir. (2.16) eşitliğinde $r$ yerine $r z$ yazılırsa,

$$
[f(z), z] r z f(z)=0, \forall r, z \in R
$$

sağlanır.Şimdi (2.16) eşitliği sağdan $z \in R$ ile çarpılırsa

$$
[f(z), z] r f(z) z=0, \forall r, z \in R
$$

olur. Son iki eşitlik birbirinden çıkarılarak düzenlenirse

$$
[f(z), z] r[f(z), z]=0, \forall z, r \in R
$$

elde edilir. $R$ yarı-asal halka olduğu için

$$
[f(z), z]=0, \forall z \in R
$$

olur. Böylece Lemma 2.3 den $R$ halkası sıfırdan farklı merkezil bir ideal kapsar. 


\section{KAYNAKLAR}

[1]. Ashraf, M. ve Rehman, N. (2001). On derivations and commutativity in prime rings: East-West J. of Math., 3 (1), 87-91.

[2]. Ashraf, M., Asma, A. ve Shakir, A. (2007). Some commutativity theorems for rings generalized derivations: Southeast Asian Bulletin of Math., 31, 415-421.

[3]. Bell, H. E., Martindale III, W. S., (1988). On semi-derivations and commutativity in prime rings: Canadian Math. Bull., 31.(4), 500-508.

[4]. Bell, H. E. and Kappe, L. C. (1989). Rings in which derivations satisfy certain algebraic conditions: Acta Math. Hungarica, 53, 339-346.

[5]. Bergen, J., (1983). Derivations in prime rings: Canadian Math. Bull., 26 (3), 267-270.

[6]. Brešar, M.,Vukman, J., (1989). Orthogonal derivation and an extension of a theorem of Posner: Rad. Mat.5, no. 2, 237-246.

[7]. Bresar, M., (1990). Semi-derivations of prime rings: Proc. Amer. Math. Soc., 108 (4), 859-860.

[8]. Chang, J. C., (1984). On semi-derivations of prime rings: Chinese Journal Mathematics, 12(4), 255 262.

[9]. Nabiel, H., (2013). Semiderivations and commutativity in semiprime rings: Gen. Math. Notes, Vol. 19, No. 2, 71-82.

[10].Posner, E.C.,(1957). Derivations in prime rings: Proc. Amer. Math. Soc., 8, 1093-1100. 